\title{
Three-Dimensional Hydrodynamic Model for Prediction of Falling Cylinder Through Water Column
}

\author{
Peter C. Chu, chu@nps.navy.mil \\ Chenwu Fan, fan@,nps.navy.mil \\ Ashley Evans, ashley.evans@navy.mil \\ Anthony F. Gilles, anthony.gilles@,navy.mil \\ Naval Postgraduate School, Monterey, CA 93943 \\ Peter Fleischer, fleischerp@navo.navy.mil \\ Naval Oceanographic Office, Stennis Space Center, MS 39529
}

1

\begin{abstract}
A three dimensional hydrodynamic model based on triple coordinate systems is developed to predict translation and orientation of falling rigid cylinder through the water column: earth-fixed coordinate (E-coordinate), cylinder's main-axis following coordinate (M-coordinate), and hydrodynamic force following coordinate (Fcoordinate). Use of the triple coordinate systems and the transforms among them leads to the simplification of the dynamical system. The body and buoyancy forces and their moments are easily calculated using the E-coordinate system. The hydrodynamic forces (such as the drag and lift forces) and their moments are easily computed using the $F$ coordinate. The cylinder's moments of gyration are simply represented using the $M$-coordinate. A recursive model is developed on the base of the triple coordinate transform to predict the cylinder's translation and orientation. To evaluate the recursive model, a cylinder-drop experiment was conducted at the Naval Postgraduate School swimming pool in June 2001. Comparison between the modeled and observed results shows a great potential of using the triple coordinate transform.
\end{abstract}

Key Words - Triple coordinate systems, momentum of cylinder, moment of momentum of cylinder, cylinder drop experiment (CIDEX).

\section{INTRODUCTION}

Study on the movement of a rigid body in fluid has wide scientific significance and technical application. The theory of dynamics of a rigid cylinder allows one to set up six nonlinear equations for the most general motion: three momentum equations and three moment-ofmomentum equations. The scientific studies of the

\footnotetext{
1 This work was sponsored by the Office of Naval Research (N0001401WR20218) and the Naval Oceanographic Office (N6230600PO00005).
}

hydrodynamics of a rigid cylinder in fluid involve the nonlinear dynamics, flight theory, body-fluid interaction, and instability theory. The body forces include the gravity and the buoyancy force. The hydrodynamic forces include the drag and lift forces that depend on the fluid-to-body velocity. Usually, a nonlinear dynamical system is needed to predict a falling rigid body in fluid, e.g., von Mises [1].

When the rigid body is cylindrical, this dynamical system can be simplified using three coordinate systems: earth-fixed coordinate (E-coordinate), cylinder's mainaxis following coordinate (M-coordinate), and hydrodynamic force following coordinate (F-coordinate). The origin of both $\mathrm{M}$ - and F-coordinates is at the cylinder's COM. The body forces and their moments are easily calculated using the E-coordinate system. The hydrodynamic forces and their moments are easily computed using the F-coordinate. The cylinder's moments of gyration are simply represented using the Mcoordinate. In this study, a recursive model is established to predict the cylinder's translation velocity and orientation on the base of the triple coordinate transformation, e.g., Crane and Duffy [2].

To evaluate the recursive model, a cylinder-drop experiment was conducted at the Naval Postgraduate School swimming pool in June 2001. It consisted of dropping cylinders into the water and recording the position, orientation, and time using two digital cameras at $(30 \mathrm{~Hz})$ as the cylinders fell 2.4 meters to the pool bottom.

Theoretical model development and cylinder drop experiment are depicted in this paper. The outline of this paper is as follows: A description of the dynamics of falling cylinder is given in Section 2. A depiction of the model cylinders and Cylinder Drop Experiment is given Sections 3 and 4. Experimental data retrieval and analysis are given in Section 5. The experimental results are described in Section 6. Model-data inter-comparison 
is presented in Section 7. The conclusions are listed in Section 8.

\section{DYNAMICS}

\section{A. Three Coordinate Systems}

Consider an axially symmetric cylinder with the centers of mass $(\mathbf{X})$ and volume (B) on the main axis (Fig. 1). Let $(L, d, \chi)$ represent the cylinder's length, diameter, and the distance between the two points $(\mathbf{X}, \mathbf{B})$. The positive $\chi$-values refer to nose-down case, i.e., the $\mathrm{COM}$ is lower than the COV. Three coordinate systems are used to model the hydrodynamics of falling cylinder through the water column: E-, M-, and F-coordinate systems. All the systems are three-dimensional, orthogonal, and right-handed. The benefit is to simplify the following computations: the body and buoyancy forces and their moments in the E-coordinate system, the hydrodynamic forces (such as the drag and lift forces) and their moments in the F-coordinate, and the cylinder's moments of gyration in the M-coordinate.

The E-coordinate system is fixed to the Earth surface with horizontal sides as $x$ and $y$-axes (along the two sides of the pool), and vertical direction as $z$-axis (upward positive, Fig. 2). Suppose a cylinder falling into the water column. The cylinder rotates around its main axis $\left(r_{1}\right)$ with an angle $\psi_{1}$ and an angular velocity of $\Omega$. Its position is represented by the center of mass (COM), and its orientation is represented by two angles: $\psi_{2}$ and $\psi_{3}$

(Fig. 3). Here, $\psi_{2}$ is the angle between the $r_{1}$-axis and the horizontal plane; and $\psi_{3}$ is the angle between the projection of the main axis in the $(x, y)$ plane and the $x$ axis. The angle, $\psi_{2}+\pi / 2$, is usually called attitude.

The relative coordinate is rigidly connected with the cylinder. The origin $(O)$ of the relative coordinate system coincides with the center of mass (COM); the $r_{1}$-axis is along the central line of the cylinder; the $r_{2}$-axis is perpendicular to the plane constructed by $r_{1}$-axis and $z$ axis ( $r_{1}-\mathrm{z}$ plane); and the $r_{3}$-axis lies in the $\left(r_{1}-z\right)$ plane and is perpendicular to $r_{1}$-axis. The selection of axes $(x$, $y, z)$ and $\left(r_{1}, r_{2}, r_{3}\right)$ follows the right-hand rule. Let $\mathbf{V}^{*}=$ $\left(V_{1}^{*}, V_{2}^{*}, V_{3}^{*}\right)$ be the three components of the velocity of COM, i.e., be the origin velocity of the coordinate system $\left(r_{1}, r_{2}, r_{3}\right)$. The geometric center (GC) is located at $(\chi, 0,0)$. For $\mathrm{GC}$ below COM, $\chi>0$, and for GC above COM, $\chi<0$. The relative coordinate system $\left(r_{1}\right.$, $\left.r_{2}, r_{3}\right)$ is obtained through the translation and two rotations $\psi_{2}$ and $\psi_{3}$ of the earth coordinate system. Let the position vector $\mathbf{P}$ be represented by $\mathbf{P}_{\mathbf{E}}$ and $\mathbf{P}_{\mathbf{B}}$ in the earth and relative coordinate systems, $\mathbf{P}_{\mathbf{E}}$ and $\mathbf{P}_{\mathbf{B}}$ are connected by

$$
\mathbf{P}_{\mathrm{E}}=\left[\begin{array}{ccc}
\cos \psi_{3} & -\sin \psi_{3} & 0 \\
\sin \psi_{3} & \cos \psi_{3} & 0 \\
0 & 0 & 1
\end{array}\right]\left[\begin{array}{ccc}
\cos \psi_{2} & 0 & \sin \psi_{2} \\
0 & 1 & 0 \\
-\sin \psi_{2} & 0 & \cos \psi_{2}
\end{array}\right] \mathbf{P}_{\mathrm{B}}+\left[\begin{array}{c}
x_{m}^{*} \\
y_{m}^{*} \\
z_{m}^{*}
\end{array}\right]
$$

where $\left(x_{\mathrm{m}}{ }^{*}, y_{\mathrm{m}}{ }^{*}, z_{\mathrm{m}}{ }^{*}\right)$ represent the position of COM in the earth coordinate system.

\section{B. Nonlinear Dynamical Equations}

Any solid object falling through a fluid (air and water) should obey two physical principles: (1) momentum balance and (2) moment of momentum balance. Let $\mathbf{V}_{\mathrm{w}}=$ $\left(V_{\mathrm{w} 1}, V_{\mathrm{w} 2}, V_{\mathrm{w} 3}\right)$ be the water velocity, and $\left(\omega_{1}^{*}, \omega_{2}^{*}, \omega_{3}^{*}\right)$ be the components of the angular velocity, referring to the direction of the relative coordinate system. The independent and dependent variables are made nondimensional by:

$$
t=\sqrt{\frac{g}{L}} t^{*}, \quad \omega=\sqrt{\frac{L}{g}} \omega^{*}, \quad \mathbf{V}=\frac{\mathbf{V}^{*}}{\sqrt{g L}},
$$

where $g$ is the gravitational acceleration, and $L$ the length of the cylinder. The non-dimensional momentum equations for COM are given by (Mises 1959)

$$
\begin{gathered}
\frac{d V_{1}}{d t}+\omega_{2} V_{3}-\omega_{3} V_{2}=\frac{\rho-\rho_{w}}{\rho} \sin \psi_{2}+\frac{F_{1}^{*}}{\rho g \Pi}, \\
\frac{d V_{2}}{d t}+\omega_{3} V_{1}=\frac{F_{2}^{*}}{\rho g \Pi}, \\
\frac{d V_{3}}{d t}-\omega_{2} V_{1}=-\frac{\rho-\rho_{w}}{\rho} \cos \psi_{2}+\frac{F_{3}^{*}}{\rho g \Pi},
\end{gathered}
$$

where $\Pi$ is the volume of the cylinder, $\rho$ the cylinder density, $\rho_{w}$ the water density, $\left(F_{1}^{*}, F_{2}{ }^{*}, F_{3}{ }^{*}\right)$ the components of water drag. The non-dimensional equations of the moment of momentum for axial symmetric cylinder are

$$
\begin{aligned}
& \frac{d \Omega}{d t}+\frac{J_{3}-J_{2}}{J_{1}} \omega_{2} \omega_{3}=\frac{L M_{1}^{*}}{g J_{1}}, \\
& \frac{d \omega_{2}}{d t}=-\frac{\chi^{*} \Pi\left(\rho-\rho_{w}\right) L}{J_{2}} \cos \psi_{2}+\frac{L M_{2}^{*}}{g J_{2}}, \\
& \frac{d \omega_{3}}{d t}=\frac{L M_{3}^{*}}{g J_{3}},
\end{aligned}
$$

where $\chi^{*}$ is the distance between COM and geometric center (GC), $\left(M_{1}^{*}, M_{2}^{*}, M_{3}^{*}\right)$ the components of the 
moment due to water drag, $\left(J_{1}, J_{2}, J_{3}\right)$ the three moments of gyration,

$$
\begin{aligned}
& J_{1}=\int\left(r_{2}^{2}+r_{3}^{2}\right) d m^{*}, \quad J_{2}=\int\left(r_{3}^{2}+r_{1}^{2}\right) d m^{*}, \\
& J_{3}=\int\left(r_{1}^{2}+r_{2}^{2}\right) d m^{*} .
\end{aligned}
$$

The orientation of the cylinder $\left(\psi_{2}, \psi_{3}\right)$ is determined by

$$
\frac{d \psi_{2}}{d t}=\omega_{2}, \quad \cos \psi_{2} \frac{d \psi_{3}}{d t}=\omega_{3} .
$$

The eight non-dimensional nonlinear equations (2a-c), (3a-c), and (5) are the basic system for determining the cylinder movement in the water column. Chu et al. [3] developed a triple coordinate transform method to solve the dynamical equations (3)-(5). The model outputs are compared to the experimental data collected during the Cylinder Drop Experiment (CYDEX) conducted at the NPS swimming pool in June 2002.

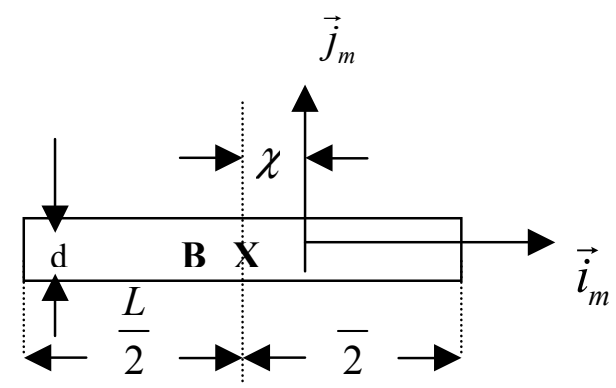

Fig. 1. M-coordinate with the COM as the origin $X$ and $\left(i_{m}\right.$, $\mathrm{j}_{\mathrm{m}}$ ) as the two axes. Here, $\chi$ is the distance between the $\operatorname{COV}(\mathrm{B})$ and $\operatorname{COM},(L, d)$ are the cylinder's length and diameter.

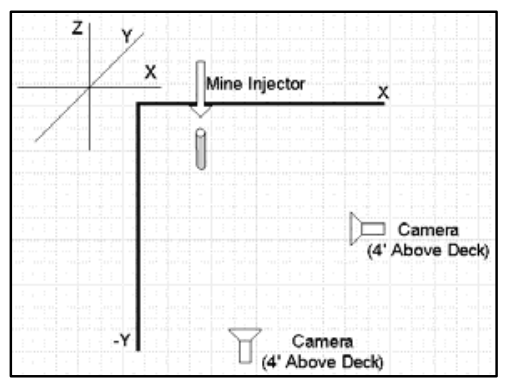

Figure 2. Earth coordinate system.

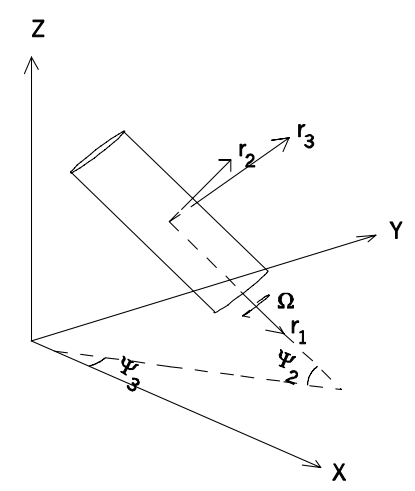

Figure 3. Cylinder orientation and relative coordinate system.

\section{MODEL CYLINDERS}

\section{A. Description}

Three model cylinders were used for the drop experiment at the Naval Postgraduate School swimming pool. All had a circular diameter of $4 \mathrm{~cm}$, however the lengths were 15, 12 and $9 \mathrm{~cm}$ respectively (Chu et al. [5]). The bodies were constructed of rigid plastic with aluminum-capped ends. Inside each was a threaded bolt, running lengthwise across the cylinder, and an internal weight (Fig. 4). The internal cylindrical weight made by copper was used to vary the cylinder's center of mass and could be adjusted fore or aft. The center of gravity of the model cylinder is the origin of the body fixed coordinate system.

The model cylinder is composed of six uniform cylindrical parts (Fig. 4): (a) a plastic hollow cylinder $C^{(1)}$ with mass of $m_{1}$, outer and inner radii of $R_{1}$ and $R_{2}$, length of $\left(L-2 l_{1}\right)$, and the center of gravity for the part (COMP) to be at its geometric center located along the $r_{1}$-axis at $r_{1}=\chi$; (b) an aluminum-capped left end (Fig. 5) solid cylinder $\mathrm{C}^{(2)}$ with mass of $m_{2}$, radius of $R_{1}$, length of $l_{1}$, and COMP located along the $r_{1}$-axis at $r_{1}=$ $L / 2-l_{1} / 2+\chi$; (c) an aluminum-capped right end solid cylinder $\mathrm{C}^{(3)}$ with mass of $m_{3}$, radius of $R_{1}$, length of $l_{1}$, and COMP located along the $r_{1}$-axis at $r_{1}=L / 2-l_{1} / 2-\chi$;

(d) a cylindrical thread $\mathrm{C}^{(4)}$ with mass of $m_{4}$, radius of $R_{3}$, length of $\left(L-2 l_{1}\right)$, and COMP located along the $r_{1}$-axis at $r_{1}=\chi$; (e) a cylindrical threaded bolt $\mathrm{C}^{(5)}$ with mass of $m_{5}$, out and inner radii of $R_{2}$ and $R_{3}$, length of $l_{2}$, and COMP located along the $r_{1}$-axis at $r_{1}=L / 2-\chi-l_{1}-l_{2} / 2$; (f) an adjustable copper cylindrical weight $\mathrm{C}^{(6)}$ with mass of m6, outer and inner radii of $R_{2}$ and $R_{3}$, length of $l_{3}$, and COMP located along the $r_{1}$-axis at $\mathrm{r} 1=\delta+\chi$, where $\delta$ is the distance between the COMP of the adjustable weight and the geometric center of the model cylinder. 


\section{B. Moments of Gyration}

Since the six parts (all cylinders) all have uniform mass distribution, the moments of gyration for these parts are:

$$
\begin{aligned}
& J_{1}^{(1)}=\frac{m_{1}}{2}\left(R_{1}^{2}+R_{2}^{2}\right), \quad J_{1}^{(2)}=\frac{m_{2}}{2} R_{1}^{2}, \quad J_{1}^{(3)}=\frac{m_{3}}{2} R_{1}^{2}, \\
& J_{1}^{(4)}=\frac{m_{4}}{2} R_{3}^{2}, \quad J_{1}^{(4)}=\frac{m_{4}}{2} R_{3}^{2}, J_{1}^{(6)}=\frac{m_{6}}{2}\left(R_{2}^{2}+R_{3}^{2}\right), \\
& J_{2}^{(1)}=J_{3}^{(1)}=\frac{m_{1}}{12}\left[3 R_{1}^{2}+3 R_{2}^{2}+\left(L-2 l_{1}\right)^{2}\right], \\
& J_{2}^{(2)}=J_{3}^{(2)}=\frac{m_{2}}{12}\left(3 R_{1}^{2}+l_{1}^{2}\right), \quad J_{2}^{(3)}=J_{3}^{(3)}=\frac{m_{3}}{12}\left(3 R_{1}^{2}+l_{1}^{2}\right), \\
& J_{2}^{(4)}=J_{3}^{(4)}=\frac{m_{4}}{12}\left[3 R_{3}^{2}+\left(L-2 l_{1}\right)^{2}\right], \\
& J_{2}^{(5)}=J_{3}^{(5)}=\frac{m_{5}}{12}\left(3 R_{2}^{2}+3 R_{3}^{2}+l_{2}^{2}\right), \\
& J_{2}^{(6)}=J_{3}^{(6)}=\frac{m_{6}}{12}\left(3 R_{2}^{2}+3 R_{3}^{2}+l_{3}^{2}\right),
\end{aligned}
$$

where the superscripts for the moments indicate the cylindrical parts. The resultant moments of gyration is computed by

$$
\begin{aligned}
& J_{1}=\sum_{j=1}^{6} J_{1}^{(j)}, \\
& J_{2}=J_{3}=\sum_{j=1}^{6} J_{2}^{(j)}+m_{1} \chi^{2}+m_{2}\left(\frac{L-l_{1}}{2}-\chi\right)^{2}+m_{3}\left(\frac{L-l_{1}}{2}+\chi\right)^{2} \\
& +m_{4} \chi^{2}+m_{5}\left(\frac{L}{2}-\chi-l_{1}-\frac{l_{2}}{2}\right)^{2}+m_{6}(\delta+\chi)^{2} .
\end{aligned}
$$

According to the definition of COM, the coordinate of $\mathrm{GC}(\chi)$ is determined by

$$
\chi=\frac{\left[m_{5}\left(L / 2-l_{1}-l_{2} / 2\right)-m_{6} \delta\right]}{\sum_{j=1}^{6} m_{j}},
$$

which indicates how the adjustable weight determines the location of COM for the model cylinder.

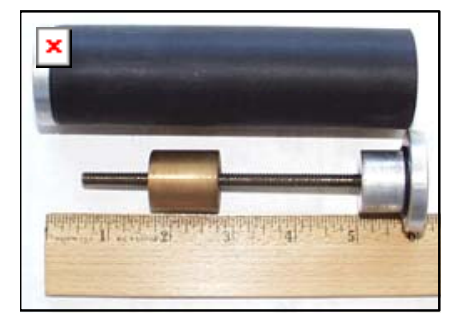

Figure 4. Internal components of the model cylinder.

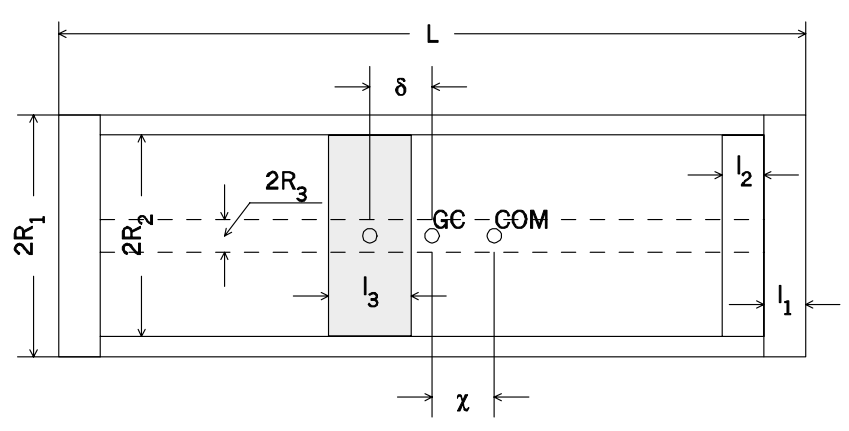

Figure 5. Internal structure of the model cylinder.

\section{A. Model Parameters}

\section{C.1. Aspect and Density Ratios}

Our goal was to choose a scale that was somewhat representative of the real world ratio of water depth to cylinder length, but at the same time would be large enough to film and would not damage the pool's bottom. The model cylinders were based on the realistic assumption that a $3 \mathrm{~m}$ cylinder is laid in water depths of $45 \mathrm{~m}$, thus producing a 15:1 ratio. The depth of the pool is $2.4 \mathrm{~m}$. From this ratio, the length $(L)$ of the model cylinder is chosen as $15 \mathrm{~cm}$. The addition of a 12 and 9 $\mathrm{cm}$ length allowed for later comparison of the sensitivity of water phase trajectory to the ratio of cylinder length over diameter. The outer radius of the model cylinder is $2 \mathrm{~cm}$. The length/diameter ratios $(L / D)$ are 15/4, 12/4, and $9 / 4$. The corresponding density ratios $\left(\rho / \rho_{w}\right)$ are $1.70,1.68$, and 1.88 , respectively.

\section{C2. $\chi$-Value}

In each of the three model cylinders, the location of the weight (i,e., the value of $\delta$ ) is adjustable. Use of (7) location of the COM ( $\chi$-value) can be determined (Table 1). The positive $\chi$-value indicates that COM is below the geometric center, and the negative $\chi$-value indicates that COM is above the geometric center.

Table 1. Model L/D, density ratio, and $\chi$-value (unit: $\mathrm{cm}$ ).

\begin{tabular}{|l|l|l|l|l|l|l|l|}
\hline $\begin{array}{l}\text { Cylinde } \\
\mathrm{r}\end{array}$ & $\mathrm{L} / \mathrm{D}$ & $\rho / \rho_{w}$ & $\chi_{1}$ & $\chi_{2}$ & $\chi_{0}$ & $\chi_{-1}$ & $\chi_{-2}$ \\
\hline 1 & $15 / 4$ & 1.70 & 1.85 & 3.69 & 0 & - & - \\
\hline 2 & $12 / 4$ & 1.68 & 1.21 & 2.43 & 0 & - & - \\
& & & & & & 1.21 & 2.43 \\
\hline 3 & $9 / 4$ & 1.88 & 0.68 & 1.37 & 0 & - & - \\
\hline
\end{tabular}




\section{CYLINDER DROP EXPERIMENT}

A cylinder drop experiment (MIDEX) was conducted at the NPS swim pool in June 2001. The purpose of the experiment is to collect data about cylinder's motion in the water column for various combinations of the model cylinder parameters. It basically consisted of dropping each of three model cylinders into the water where each drop was recorded underwater from two viewpoints. Figure 6 depicts the overall setup. The controlled parameters for each drop were: $L / \mathrm{D}$ ratio, $\chi$-value, initial velocity $\left(\mathrm{V}_{\text {in }}\right)$, and drop angle. The Earth's coordinate system is chosen with the origin at the corner of the swimming pool with the two sides as $x$ - and $y$-axes and the vertical $z$-axis. The initial injection of cylinder was in the (y,z) plane (Fig. 2).

\section{A. Initial Velocity}

Initial velocity $\left(\mathrm{V}_{\text {in }}\right)$ was calculated by using the voltage return of an infrared photo detector located at the base of the cylinder injector. The infrared sensor produced a square wave pulse when no light was detected due to blockage caused by the cylinder's passage. The length of the square wave pulse was converted into time by using a universal counter. Dividing the cylinder's length by the universal counter's time yielded $V_{\text {in }}$. The cylinders were dropped from several positions within the injector mechanism in order to produce a range of $\mathrm{V}_{\mathrm{in}}$. The method used to determine $\mathrm{V}_{\text {in }}$ required that the infrared light sensor be located above the water's surface. This distance was held fixed throughout the experiment at $10 \mathrm{~cm}$.

\section{B. Drop Angle}

The drop angle (initial $\psi_{2}^{(i n)}$ ) was controlled using the drop angle device. Five screw positions marked the $15^{\circ}, 30^{\circ}, 45^{\circ}, 60^{\circ}$, and $75^{\circ}$. The drop angles were determined from the lay of the pool walkway, which was assumed to be parallel to the water's surface. A range of drop angles was chosen to represent the various entry angles that air and surface laid cylinders exhibit. This range produced velocities whose horizontal and vertical components varied in magnitude. This allowed for comparison of cylinder trajectory sensitivity with the varying velocity components.

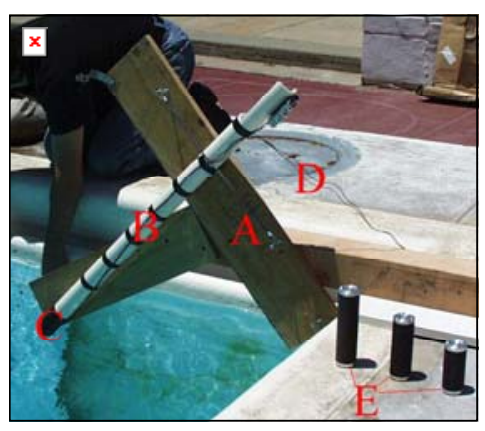

Figure 6. CIDEX equipment.

\section{Methodology}

For each run the cylinders were set to a $\chi$-value. For positive $\chi$-value, the cylinders were placed into the injector so that the COM was located below the geometric center. For negative $\chi$-value, the COM was located above the geometric center to release. A series of drops were then conducted in order of decreasing cylinder length for each angle. Table 2 indicates number of drops conducted for different drop angles and $\chi$ value for $L / D=15 / 4$. Number of drops for other $L / D$ ratios $(12 / 4,9 / 4)$ is comparable to that for $L / D$ ratio of 15/4. All together there were 712 drops. Each video camera had a film time of approximately one hour. At the end of the day, the tapes were replayed in order to determine clarity and optimum camera position.

Table 2. Number of drops conducted for different drop angles and $\chi$-values for $L / D=15 / 4$.

\begin{tabular}{|l|l|l|l|l|l|}
\hline$\psi_{2}^{(i n)}$ & $15^{\circ}$ & $30^{\circ}$ & $45^{\circ}$ & $60^{\circ}$ & $75^{\circ}$ \\
\hline$\chi_{2}$ & 13 & 15 & 15 & 15 & 12 \\
\hline$\chi_{1}$ & 9 & 15 & 15 & 15 & 9 \\
\hline$\chi_{0}$ & 12 & 14 & 15 & 18 & 6 \\
\hline$\chi_{-1}$ & 0 & 6 & 6 & 6 & 0 \\
\hline$\chi_{-2}$ & 2 & 6 & 6 & 0 & 0 \\
\hline
\end{tabular}

\section{DATA RETRIEVAL AND ANALYSIS}

\section{A. Data Retrieval}

Upon completion of the drop phase, the video from each camera was converted to digital format. The digital video for each view was then analyzed frame by frame $(30 \mathrm{~Hz})$ in order to determine the cylinder's position in the $(x, z)$ and $(y, z)$ planes. The cylinder's top and bottom positions were input into a MATLAB generated grid, similar to the ones within the pool. The first point to impact the water was always plotted first. This facilitated 
tracking of the initial entry point throughout the water column. The cameras were not time synchronized; thus, the first recorded position corresponded to when the full length of the cylinder was in view.

\section{B. Source of Errors}

There were several sources of error that hindered the determination of the cylinder's exact position within the water column. Locations above or below the camera's focal point were subjected to parallax distortion. Placing the cameras as far back as possible, while still being able to resolve the individual grid squares, minimized this error. Second, the background grids were located behind the cylinder's trajectory plane. This resulted in the cylinder appearing larger than normal. This error was minimized by not allowing the plotted points to exceed the particular cylinder's length. Third, an object injected into the water will generate an air cavity. This air cavity can greatly affect the initial motion, particularly at very high speeds (hydro ballistics). The air cavity effect was deemed to be minimal due to the low inject velocities used.

\section{Data Analysis}

The 3-D data provided by each camera was first used to produce raw two 2-D plots of the cylinder's trajectory. Next, 2-D data from both cameras was then fused to produce a 3-D history. This 3-D history was then made non-dimensional in order to generalize the results. The non-dimensional data was used to generate impact scatter plots and was also used in multiple linear regression calculations.

\section{EXPERIMENTAL RESULTS}

\section{A. Trajectory Patterns}

After analyzing the 3-D data set, seven trajectory patterns were found. The plots on the $(y, z)$ plane were chosen for trajectory analysis, as this plane was parallel to the direction of the cylinder drop. The generalized trajectory patterns are described in Table 3, and Figures 7-10. The water phase trajectory a cylinder experiences ultimately determines the impact orientation. In MIDEX, the categorizing of trajectories into general patterns served two purposes. Observed trajectories were found to be most sensitive to $\chi$-value, drop angle and $L / D$ ratio. As COM distance ( $\chi$-value) increased from GC the cylinder tended to follow a straight pattern. As COM was moved closer to the $\mathrm{GC}$ (decreasing $\chi$-value) the cylinder's trajectory tended towards being more parallel with the pool's bottom. At steep drop angles, the cylinder experienced little lateral movement and tended towards a straight pattern. Additionally, as $L / D$ ratio decreased more complex trajectory patterns developed. This included significant oscillation about the vertical axis and increased lateral movement.

Table 3. Description of relative coordinate based trajectory patterns.

\begin{tabular}{|l|l|}
\hline Trajectory Pattern & Description \\
\hline Straight (or Slant) & $\begin{array}{l}\text { Little angular change, } \\
\text { Nearly parallel to } z \text {-axis, } \\
\psi_{2} \text { near }\left(90^{\circ} \pm 15^{\circ}\right)\end{array}$ \\
\hline Spiral & Oscillating (no rotation) \\
\hline Flip & Only once as $\chi^{*}<0$ \\
\hline Flat & $\psi_{2}$ near $0^{\circ}$ (no oscillation) \\
\hline Seesaw & $\psi_{2}$ near $0^{\circ}$ (oscillation) \\
\hline Combination & Several of the above patterns \\
\hline
\end{tabular}

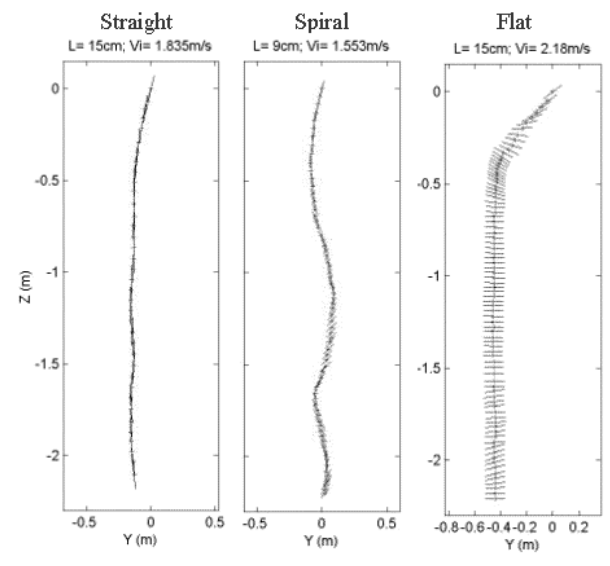

Figure 7. Trajectory patterns.
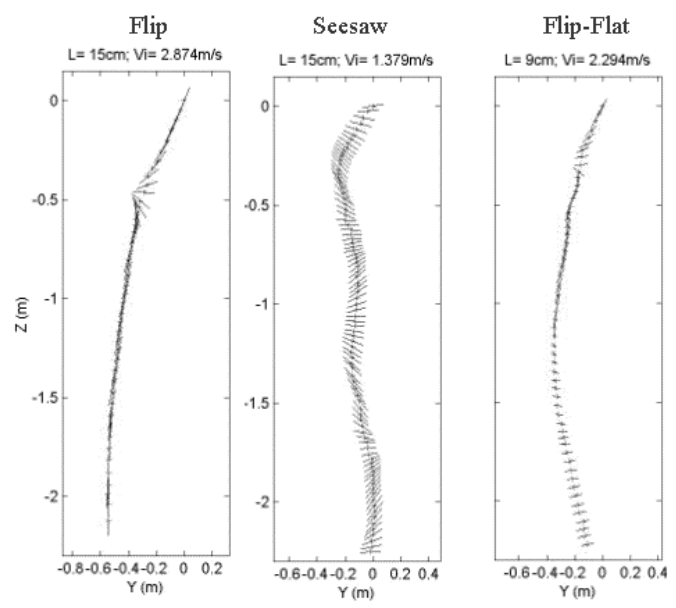

Figure 8. Trajectory patterns. 


\begin{tabular}{|l|c|c|c|}
\hline & COM Position: 2 & \multicolumn{2}{|c|}{} \\
\hline Mine Length: & 15 & 12 & 9 \\
\hline Drop Angle: $15^{\circ}$ & Straight & Straight & Straight-Slant \\
& Slant-Straight & Spiral & Spiral \\
\cline { 2 - 4 } & Slant-Straight & Slant-Straight & Slant-Straight \\
\cline { 2 - 4 } & Slant-Straight & Slant-Straight & Spiral \\
\hline Drop Angle: $30^{\circ}$ & Slant-Straight & Slant & Spiral \\
\cline { 2 - 4 } & Straight & Spiral & Spiral \\
\cline { 2 - 4 } & Slant-Straight & Straight & Spiral \\
\cline { 2 - 4 } & Slant-Straight & Slant-Straight & Spiral \\
\cline { 2 - 4 } & Slant-Straight & Slant-Straight & Spiral \\
\hline Drop Angle: $45^{\circ}$ & Slant-Straight & Spiral & Spiral \\
\cline { 2 - 4 } & Slant & Spiral & Spiral \\
\cline { 2 - 4 } & Straight-Spiral & Straight-Spiral & Spiral \\
\cline { 2 - 4 } & Straight & Straight & Spiral \\
\hline Drop Angle: $60^{\circ}$ & Slant & Slant-Straight & Slant-Spiral \\
\hline & Straight & Straight-Slant & Spiral \\
\cline { 2 - 4 } & Straight & Straight & Spiral \\
\cline { 2 - 4 } & Straight & Straight & Spiral \\
\cline { 2 - 4 } & Straight & Straight-Spiral & Straight-Spiral \\
\cline { 2 - 4 } & Straight & Straight & Spiral \\
\hline Drop Angle: $75^{\circ}$ & Straight & Straight & Spiral \\
\hline & Straight & Straight-Spiral & Slant \\
\cline { 2 - 4 } & Straight & Straight & Spiral \\
\cline { 2 - 4 } & Straight & Straight-Spiral & Straight-Spiral \\
\cline { 2 - 4 } & Straight & Straight-Spiral & Straight-Spiral \\
\hline
\end{tabular}

Figure 9. Trajectory patterns for $\chi=\chi_{2}$.

\begin{tabular}{|l|c|c|c|}
\hline & COM Position: -2 & \multicolumn{2}{|c|}{} \\
\hline Mine Length: & 15 & 12 & 9 \\
\hline Drop Angle: $30^{\circ}$ & Flip-Straight & Flip-Slant & Flip-Straight-Spiral \\
\hline & Flip-Straight & Flip-Straight & Flip-Straight-Spiral-Flip \\
\hline Drop Angle: $45^{\circ}$ & Flip-Straight & Flip-Straight-Spiral & Flip-Straight \\
\hline & Flip-Straight & Flip-Straight & Flip-Straight-Spiral \\
\cline { 2 - 2 } & COM Position: -1 & \multicolumn{2}{|c|}{} \\
\hline Drop Angle: $30^{\circ}$ & Flip-Straight & Flip-Slant & Straight-Flip-Seesaw \\
\hline & Flip-Slant & Flip-Straight & Flip-Straight-Spiral \\
\hline Drop Angle: $45^{\circ}$ & Flip-Spiral-Slant & Flip-Slant & Flip-Spiral \\
\hline & Flip-Slant & Flip-Slant & Flip-Spiral \\
\hline Drop Angle: $60^{\circ}$ & Flip-Straight & Flip-Straight & Flip-Spiral-Seesaw \\
\hline & Straight-Flip & Slant-Flip-Slant & Flip-Spiral \\
\hline
\end{tabular}

Figure 10. Trajectory patterns for negative COM position $\left(\chi_{-1}, \chi_{-2}\right)$. Here, COM is above GC when the cylinder enters the surface).

\section{B. Impact Attitude}

The angle, $\psi_{2}+\pi / 2$, is the impact attitude at the bottom of the water column. The cylinder burial is largely determined from the impact attitude of the cylinder. Cylinders whose impact attitudes are perpendicular $\left(\psi_{2}=90^{\circ}\right)$ to the sediment interface will experience the largest degree of impact burial (Taber 1999). It is therefore important to analyze the relationship between impact attitude and the controlled parameters, drop angle, $V_{\text {in }}, L / D$, and $\chi$. The experiment shows that both $L / D$ and $V_{\text {in }}$ had little influence on impact attitude. The drop angle and $\chi$, however, were the largest determinants of impact attitude.

From Figure 11 it is apparent that there are several peaks centered near $90^{\circ}, 140^{\circ}$, and $180^{\circ}$. Further analysis reveals that these peaks correspond to the COM positions 0,1 and 2 (corresponding to $\chi_{0}, \chi_{1}, \chi_{2}$ ), respectively.

COM positions -1 and -2 (corresponding to $\chi_{-1}, \chi_{-2}$ ) followed the same trend as their positive counterparts.

Although drop angle was not the most influential parameter, variations did induce changes in impact orientation. As drop angle increased, the likelihood of any lateral movement decreased. This allowed for impact angles that were more vertically orientated. This is primarily due to the fact that the vertical components of velocity were greater than those at shallow angles. Thus, the time to bottom and time for trajectory alteration was less.

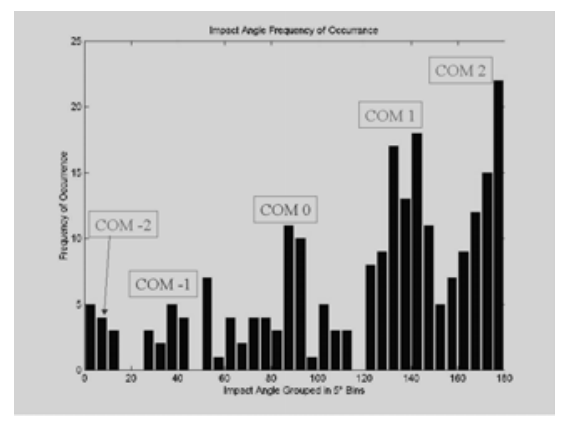

Figure 11. Relationship between COM position and impact attitude.

\section{MODEL-EXPERIMENT COMPARISON}

The 3D model predicts the motion of cylinder inside the water column. The drop angle and velocity in CYDEX are not the initial conditions for the recursive model. For each drop in CYDEX, the undersea cameras measure the initial translation, velocity, orientation, and angular velocity. The model-experiment comparison starts just after the cylinders are fully immersed into the water column. The model well predicts the cylinder's motion. Here, we show some examples. We plan to verify the model using NRL data (Holland et al. [6]).

\subsection{Positive $\chi$ (Nose-Down)}

\section{Example-1}

Cylinder \#1 $\left(L=15.20 \mathrm{~cm}, \bar{\rho}=1.69 \mathrm{~g} \mathrm{~cm}^{-3}\right)$ with $\chi=0.74 \mathrm{~m}$ is injected to the water with the drop angle $45^{\circ}$. The physical parameters of this cylinder are given by

$$
J_{1}=330.5 \mathrm{~g} \mathrm{~cm}^{2}, J_{2}=J_{3}=5783.0 \mathrm{~g} \mathrm{~cm}^{2} .
$$

Undersea cameras measure the initial conditions

$$
\begin{aligned}
& x_{0}=0, y_{0}=0, z_{0}=0, \\
& u_{0}=0, v_{0}=-1.55 \mathrm{~m} \mathrm{~s}^{-1}, w_{0}=-2.52 \mathrm{~m} \mathrm{~s}^{-1}, \\
& \psi_{10}=0, \psi_{20}=60^{\circ}, \psi_{30}=-95^{\circ} \\
& \omega_{10}=0, \omega_{20}=0.49 \mathrm{~s}^{-1}, \omega_{30}=0.29 \mathrm{~s}^{-1} .
\end{aligned}
$$

Substitution of the model parameters (9a) and the initial conditions (9b) into the three-dimensional hydrodynamic model leads to the prediction of the cylinder's translation and orientation that are compared with the data collected during CYDEX at time steps (Fig. 12). Both model 
simulated and observed tracks show a slant-straight pattern.

\section{Example-2:}

Cylinder \#2 $\left(L=12.10 \mathrm{~cm}, \bar{\rho}=1.67 \mathrm{~g} \mathrm{~cm}^{-3}\right)$ with $\chi=0.53 \mathrm{~m}$ is injected to the water with the drop angle $45^{\circ}$. The physical parameters of this cylinder are given by

$$
J_{1}=271.3 \mathrm{~g} \mathrm{~cm}^{2}, J_{2}=J_{3}=3206.5 \mathrm{~g} \mathrm{~cm}^{2} .
$$

Undersea cameras measure the initial conditions

$$
\begin{aligned}
& x_{0}=0, y_{0}=0, z_{0}=0, \\
& u_{0}=0, v_{0}=-1.07 \mathrm{~m} \mathrm{~s}^{-1}, w_{0}=-1.74 \mathrm{~m} \mathrm{~s}^{-1}, \\
& \psi_{10}=0, \psi_{20}=64^{\circ}, \psi_{30}=-98^{\circ}, \\
& \omega_{10}=0, \omega_{20}=4.50 \mathrm{~s}^{-1}, \omega_{30}=-0.21 \mathrm{~s}^{-1} .
\end{aligned}
$$

The predicted cylinder's translation and orientation are compared with the data collected during CYDEX at time steps (Fig. 13). The simulated and observed tracks show straight-spiral pattern.

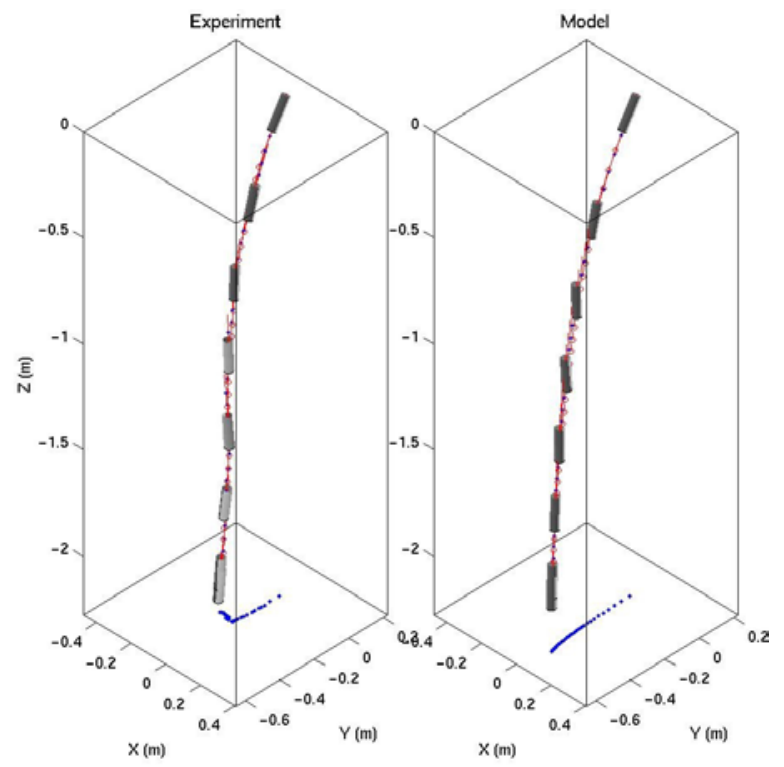

Fig. 12. Movement of Cylinder \#1 $(L=15.20 \mathrm{~cm}$, $\bar{\rho}=1.69 \mathrm{~g} \mathrm{~cm}^{-3}$ ) with $\chi=0.74 \mathrm{~m}$ and drop angle $45^{\circ}$ obtained from (a) experiment, and (b) recursive model.

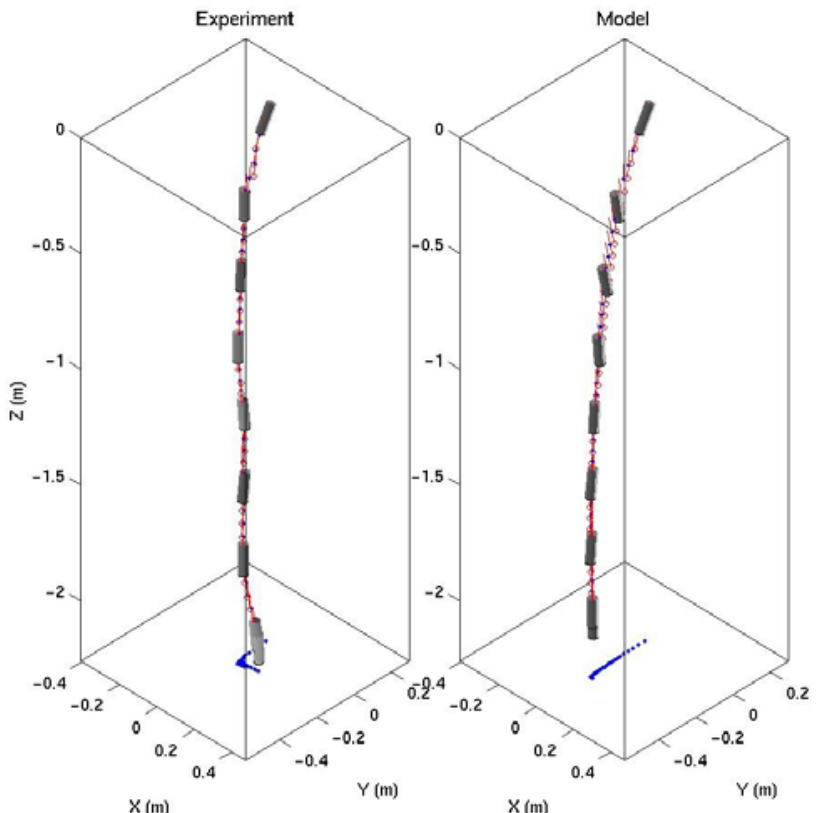

Fig. 13. Movement of Cylinder \#2 $(L=12.10 \mathrm{~cm}$, $\bar{\rho}=1.67 \mathrm{~g} \mathrm{~cm}^{-3}$ ) with $\chi=0.53$ and drop angle $45^{\circ}$ obtained from (a) experiment, and (b) recursive model.

\section{Example-3:}

Cylinder \#3 $\left(L=9.12 \mathrm{~cm}, \bar{\rho}=1.88 \mathrm{~g} \mathrm{~cm}^{-3}\right)$ with $\chi=$ $0.58 \mathrm{~m}$ is injected to the water with the drop angle $45^{\circ}$. The physical parameters of this cylinder are given by

$$
J_{1}=235.0 \mathrm{~g} \mathrm{~cm}^{2}, J_{2}=J_{3}=1556.8 \mathrm{~g} \mathrm{~cm}^{2} \text {. }
$$

Undersea camera measure the initial conditions

$$
\begin{aligned}
& x_{0}=0, y_{0}=0, z_{0}=0, \\
& u_{0}=0, v_{0}=-0.70 \mathrm{~m} \mathrm{~s}^{-1}, w_{0}=-1.31 \mathrm{~m} \mathrm{~s}^{-1}, \\
& \psi_{10}=0, \psi_{20}=64^{\circ}, \psi_{30}=-90^{\circ} \\
& \omega_{10}=0, \omega_{20}=3.96 \mathrm{~s}^{-1}, \omega_{30}=0 .
\end{aligned}
$$

The predicted cylinder's translation and orientation are compared with the data collected during CYDEX at time steps (Fig. 14). Both model simulated and observed tracks show a spiral pattern. 


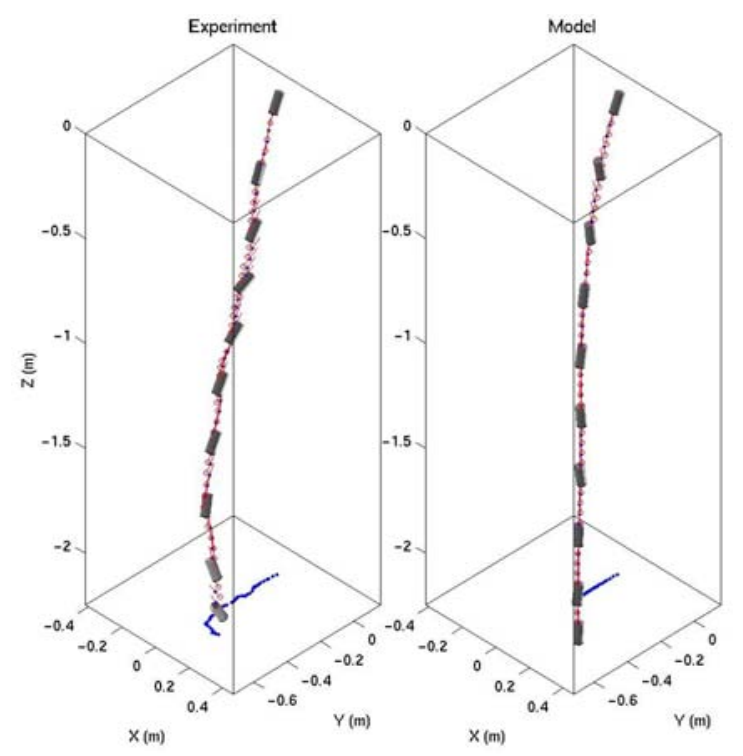

Fig. 14. Movement of Cylinder $\# 3(L=9.12 \mathrm{~cm}$, $\bar{\rho}=1.88 \mathrm{~g} \mathrm{~cm}^{-3}$ ) with $\chi=0.58 \mathrm{~m}$ and drop angle $\mathbf{4 5}^{\circ}$ obtained from (a) experiment, and (b) recursive model.

\subsection{Negative $\chi$ (Nose-Up)}

\section{Example-4:}

Cylinder \#1 $\left(L=15.2 \mathrm{~cm}, \bar{\rho}=1.69 \mathrm{~g} \mathrm{~cm}^{-3}\right)$ with $\chi=$ $-1.48 \mathrm{~cm}$ is injected to the water with the drop angle $30^{\circ}$. The physical parameters of this cylinder are given by

$$
J_{1}=330.5 \mathrm{~g} \mathrm{~cm}^{2}, J_{2}=J_{3}=6233.8 \mathrm{~g} \mathrm{~cm}^{2} .
$$

Undersea cameras measure the initial conditions

$$
\begin{aligned}
& x_{0}=0, y_{0}=0, z_{0}=0, \\
& u_{0}=0, v_{0}=-0.91 \mathrm{~m} \mathrm{~s}^{-1}, w_{0}=-0.78 \mathrm{~m} \mathrm{~s}^{-1}, \\
& \psi_{10}=0, \psi_{20}=17^{o}, \psi_{30}=-96^{o} \\
& \omega_{10}=0, \omega_{20}=-4.56 \mathrm{~s}^{-1}, \omega_{30}=0.49 \mathrm{~s}^{-1}
\end{aligned}
$$

The predicted cylinder's translation and orientation are compared with the data collected during CYDEX at time steps (Fig. 15). The simulated and observed tracks show flip-spiral pattern. The flip occurs $0.10 \mathrm{~s}(0.12 \mathrm{~s})$ after cylinder entering the water in the experiment (model). After the flip, the cylinder spirals down to the bottom.

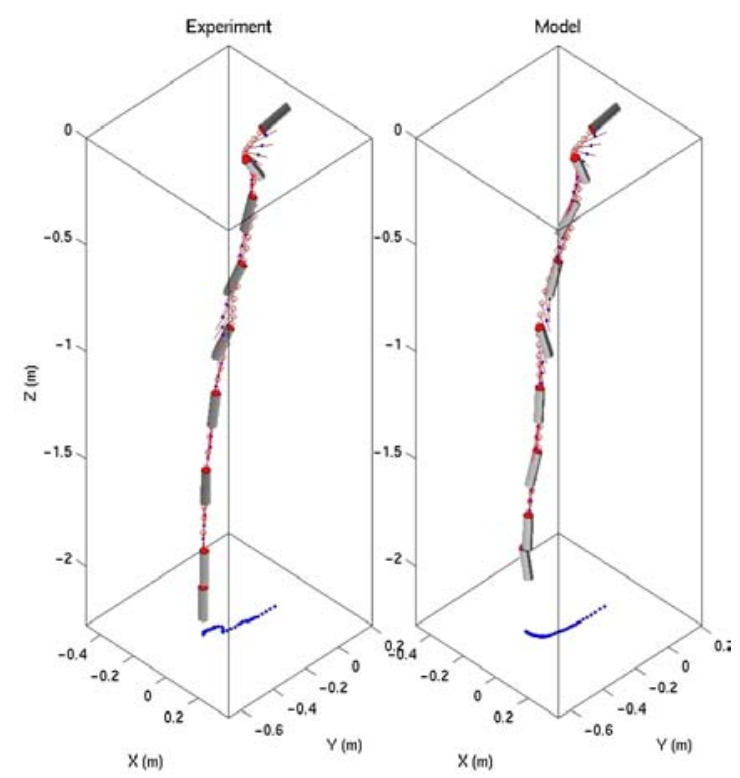

Fig. 15. Movement of Cylinder \#1 $(L=15.2 \mathrm{~cm}$, $\bar{\rho}=1.69 \mathrm{~g} \mathrm{~cm}^{-3}$ ) with $\chi=-\mathbf{- 1 . 4 8} \mathrm{cm}$ and drop angle $\mathbf{3 0}^{\circ}$ obtained from (a) experiment, and (b) recursive model.

\section{Example-5:}

Cylinder \#2 $\left(L=12.10 \mathrm{~cm}, \bar{\rho}=1.67 \mathrm{~g} \mathrm{~cm}^{-3}\right)$ with $\chi=-1.00 \mathrm{~cm}$ is injected to the water with the drop angle $30^{\circ}$. The physical parameters of this cylinder are given by

$$
J_{1}=271.3 \mathrm{~g} \mathrm{~cm}^{2}, J_{2}=J_{3}=3312.6 \mathrm{~g} \mathrm{~cm}^{2} .
$$

Undersea cameras measure the initial conditions

$$
\begin{aligned}
& x_{0}=0, y_{0}=0, z_{0}=0, \\
& u_{0}=0, v_{0}=-0.75 \mathrm{~m} \mathrm{~s}^{-1}, w_{0}=-0.67 \mathrm{~m} \mathrm{~s}^{-1}, \\
& \psi_{10}=0, \psi_{20}=24^{o}, \psi_{30}=-96^{o} \\
& \omega_{10}=0, \omega_{20}=-5.08 \mathrm{~s}^{-1}, \omega_{30}=0.15 \mathrm{~s}^{-1} .
\end{aligned}
$$

The predicted cylinder's translation and orientation are compared with the data collected during CYDEX at time steps (Fig. 16). The simulated and observed tracks show flip-spiral pattern. The flip occurs $0.11 \mathrm{~s}(0.13 \mathrm{~s})$ after cylinder entering the water in the experiment (model). After the flip, the cylinder spirals down to the bottom. 


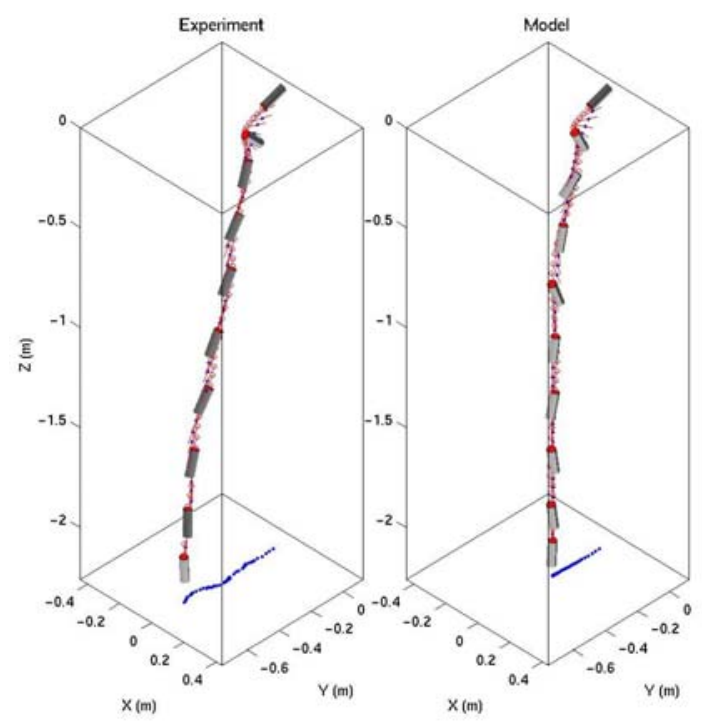

Fig. 16. Movement of Cylinder \#2 $(L=12.10 \mathrm{~cm}$, $\bar{\rho}=1.67 \mathrm{~g} \mathrm{~cm}^{-3}$ ) with $\chi=-\mathbf{1 . 0 0} \mathrm{cm}$ and drop angle $30^{\circ}$ obtained from (a) experiment, and (b) recursive model.

\subsection{Zero $\chi$ (Coincidence of COM and COV)}

\section{Example-6:}

Cylinder \#2 $\left(L=12.1 \mathrm{~cm}, \bar{\rho}=1.67 \mathrm{~g} \mathrm{~cm}^{-3}\right)$ with $\chi=$ $0 \mathrm{~cm}$ is injected to the water with the drop angle $45^{\circ}$. The physical parameters of this cylinder are given by

$$
J_{1}=271.3 \mathrm{~g} \mathrm{~cm}^{2}, J_{2}=J_{3}=3424.6 \mathrm{~g} \mathrm{~cm}^{2} \text {. }
$$

Undersea cameras measure the initial conditions

$$
\begin{aligned}
& x_{0}=0, y_{0}=0, z_{0}=0, \\
& u_{0}=0, v_{0}=-1.07 \mathrm{~m} \mathrm{~s}^{-1}, w_{0}=-1.74 \mathrm{~m} \mathrm{~s}^{-1}, \\
& \psi_{10}=0, \psi_{20}=70^{\circ}, \psi_{30}=-100^{\circ} \\
& \omega_{10}=0, \omega_{20}=-0.95 \mathrm{~s}^{-1}, \omega_{30}=0.57 \mathrm{~s}^{-1} .
\end{aligned}
$$

The predicted cylinder's translation and orientation are compared with the data collected during CYDEX at time steps (Fig. 17). The simulated and observed tracks show straight-seasaw pattern. However, the seasaw pattern occurs much earlier in the model track. Comparison among Figs. 9-14 leads to the fact that the performance of the recursive model for $\chi=0$ is not as good as for $\chi \neq 0$.

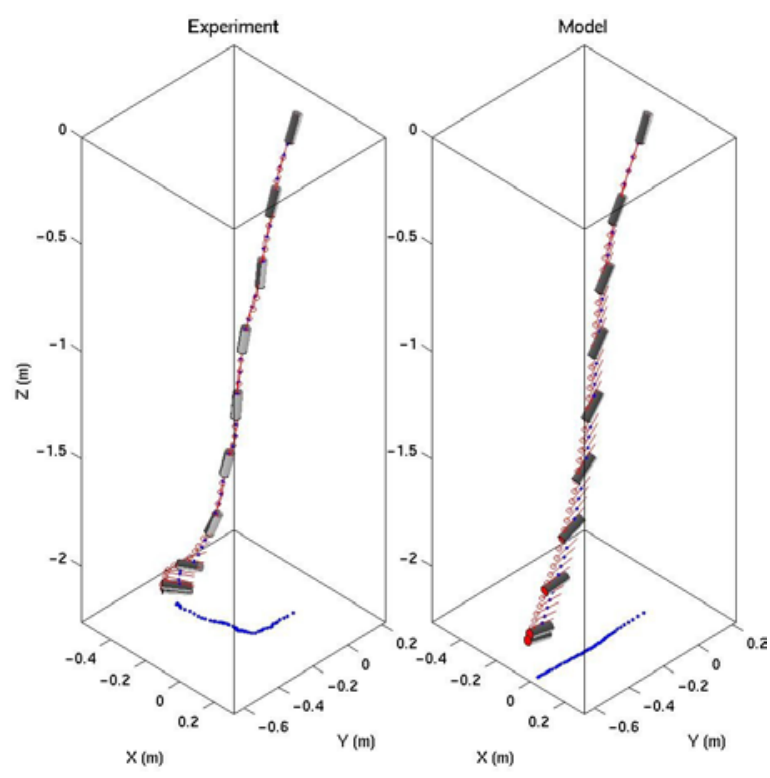

Fig. 17. Movement of Cylinder \#2 $(L=12.1 \mathrm{~cm}$, $\bar{\rho}=1.67 \mathrm{~g} \mathrm{~cm}^{-3}$ ) with $\chi=0 \mathrm{~cm}$ and drop angle $\mathbf{4 5}^{\circ}$ obtained from (a) experiment, and (b) recursive model.

\section{CONCLUSIONS}

(1) Triple coordinate systems are suggested to predict the translation and orientation of falling rigid cylinder through water column: earth-fixed coordinate (Ecoordinate), cylinder's main-axis following coordinate (M-coordinate), and hydrodynamic force following coordinate (F-coordinate). The benefit is to simplify the following computations: the body and buoyancy forces and their moments in the E-coordinate system, the hydrodynamic forces (such as the drag and lift forces) and their moments in the F-coordinate, and the cylinder's moments of gyration in the M-coordinate.

(2) Usually, the momentum (moment of momentum) equation for predicting the cylinder's translation velocity (orientation) is represented in the E-coordinate (Mcoordinate) system. Transformations among the three coordinate systems are used to convert the forcing terms into E-coordinate (M-coordinate) for the momentum (moment of momentum) equation. A recursive model is developed on the base of the triple coordinate transform to predict the cylinder's translation and orientation.

(3) The Cylinder Drop Experiment (CYDEX) was conducted at the Naval Postgraduate School (NPS) in July 2001 to evaluate the recursive model. It consisted of dropping cylinders into the water and recording the position as a function of time using two digital cameras at $(30 \mathrm{~Hz})$ as the cylinders fell 2.4 meters to the pool bottom. After analyzing the CODEX experimental data, seven general trajectory patterns are identified: straight, slant, spiral, flip, flat, see-saw, and combination. 
(4) Model-experiment comparison shows that the model well predicts the cylinder's translation and orientation. However, the performance of the recursive model for $\chi=0$ is not as good as for $\chi \neq 0$.

\section{ACKNOWLEDGMENTS}

This work was supported by the Office of Naval Research Marine Geology Program (N0001401WR20218) and the Naval Oceanographic Office (N6230600PO00005).

\section{REFERENCES}

[1] Von Mises, R, 1959, Theory of Flight, $1^{\text {st }}$ Ed., Dover Publications Inc., New York, 564-585 pp.

[2] Crane, C.D. III, and J. Duffy, 1998, Kinematic Analysis of Robot Manipulators, $1^{\text {st }}$ Ed., Cambridge University Press, Cambridge, 429 pp.

[3] Crowe, C.T., J.A. Roberson, and D.F. Elger, 2001, Engineering Fluid Mechanics. $7^{\text {th }}$ Ed., John Wiley \& Sons Inc, New York, 714 pp.

[4] Chu, P.C., C.W. Fan, A. Evans, A.F. Gilless, , and P. Fleischer, 2002. Triple coordinate transform for prediction of falling cylinder in the water column. Journal of Applied in Fluid Mechanics, submitted.

[5] Chu, P.C., A.F. Gilless, C. Fan, and P. Fleischer, 2002. Hydrodynamical Characteristics of a Falling Cylinder in the Water Column. Advances in Fluid Mechanics, vol 4, edited by M. Rahman, R. Verhoeven, and C.A. Brebbia, WIT Press, Southampton, UK, 163-181.

[6] Holland, K.T., Green, A.W., Abelev, A.V., and Valent, P.J. "Parameterization of the in-water motions of falling cylinders using high-speed video" (submitted)

Peter C. Chu ( $\left.\mathrm{PhD}, 85^{\prime}\right)$ is a professor of oceanography at the Naval Postgraduate School, Monterey, CA 93943.

Ashley Evans (MA, 02) is a Navy Lieutenant Commander who obtained MA degree in Meteorology and Oceanography at the Naval Postgraduate School in September 2002.

Chenwu Fan (MA, 82')Is an oceanographer at the Naval Postgraduate School, Monterey, CA 93943.

Peter Fleischer (Ph.D, 70') is an oceanographer at the Naval Oceanographic Office, Stennis Space Center, MS 39529.

Anthony F. Gilles (MA, 01) is a Navy Lieutenant Commander who obtained MA degree in Meteorology and Oceanography at the Naval Postgraduate School in September 2001. 